\title{
Gut Microbial Dysbiosis in Heart Failure \\ - Is It a Future Therapeutic Target or Not? -
}

\author{
Tomoya Yamashita, MD, PhD; Tomohiro Hayashi, MD, PhD; \\ Naofumi Yoshida, MD; Ken-ichi Hirata, MD, PhD
}

$\mathbf{T}$ he high prevalence of patients with chronic heart failure (HF) remains related to both poor prognosis and a huge healthcare burden. ${ }^{1}$ It has been widely recognized that the pathophysiology of HF is closely connected with multi-organ systems via neurohormonal responses and systemic inflammation. ${ }^{2}$ Several concepts have been proposed to explore the underlying pathogenesis

\section{Article p1640}

of HF, but there is a strong need for more specific therapeutic options. The environment of the human gut comprises more than 1,000 bacterial species, which develop with the host from birth, and regulate multiple host

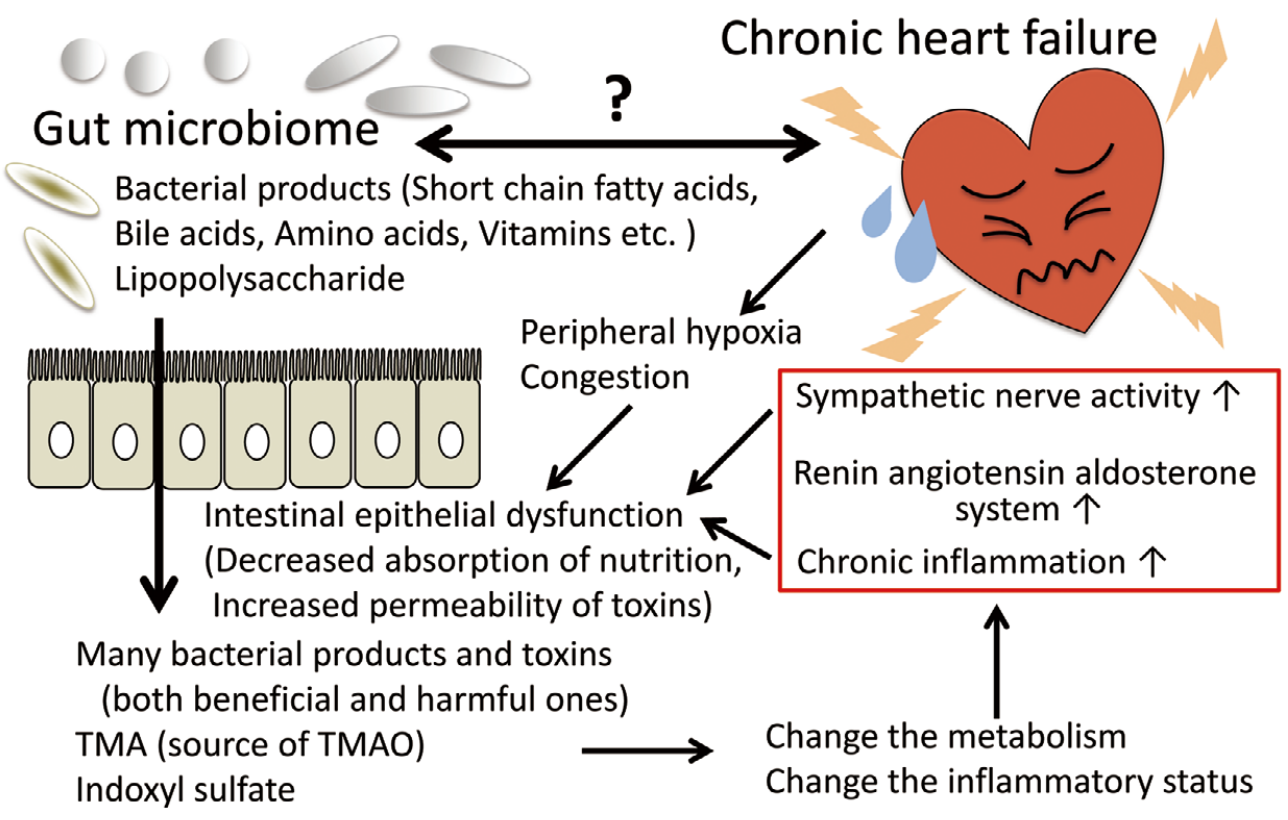

Figure. Mechanistic interaction between the gut microbiome and the pathophysiology of heart failure. In patients with chronic heart failure, increased sympathetic nerve activity, chronic inflammation, peripheral hypoxia, and congestion (edema) impair the function of the intestinal epithelium, which results in decreased absorption of nutrients and increased permeability to bacterial toxins, and thus changes in both metabolism and the inflammatory status. Nobody yet knows if the changes in the microbiome are the cause or effect of heart failure, so further research is needed to enable use of the gut microbiome composition and its functional modifications as novel diagnostic or therapeutic strategies. TMA, trimethylamine; TMAO, trimethylamine- $N$-oxide.

The opinions expressed in this article are not necessarily those of the editors or of the Japanese Circulation Society.

Received April 2, 2018; accepted April 4, 2018; released online April 24, 2018

Division of Cardiovascular Medicine, Department of Internal Medicine, Kobe University Graduate School of Medicine, Kobe, Japan

Mailing address: Tomoya Yamashita, MD, PhD, Division of Cardiovascular Medicine, Department of Internal Medicine, Kobe University Graduate School of Medicine, 7-5-1 Kusunoki-cho, Chuo-ku, Kobe 650-0017, Japan. E-mail: tomoya@med. kobe-u.ac.jp

ISSN-1346-9843 All rights are reserved to the Japanese Circulation Society. For permissions, please e-mail: cj@j-circ.or.jp 
metabolic pathways and immune responses. ${ }^{3}$ The recent development of $16 \mathrm{~S}$ ribosomal (r)RNA gene sequencing and metagenome analyses using next-generation sequencing technologies has remarkably driven the field of gut microbiome research. A deeper understanding of the interactions between the gut microbiome and host organs is a prerequisite for optimizing therapeutic strategies against metabolic and inflammatory disorders in humans. Collectively, the gut microbiome has become a focus for cardiovascular research, including $\mathrm{HF}$.

In this issue of the Journal, Katsimichas et $\mathrm{al}^{4}$ provide the results of their case-control study of sequencing the bacterial 16S rRNA genes of stool samples from nonischemic HF patients with reduced ejection fraction (HFrEF), compared with healthy control subjects. The authors recruited 18-70-year-old patients without systemic use of antibiotics, ingestion of probiotic-containing preparations, chronic kidney disease stage $4 / 5$, intestinal disorders such as inflammatory bowel disease, intestinal surgery etc. They report that these Japanese non-ischemic HF patients exhibited differences in gut microbial communities compared with healthy subjects. Gut microbiome $\alpha$ diversity (the number of microbial species) was not different, but the $\beta$ diversity (evenness of microbial composition) was altered. At the genus level, Streptococcus and Veillonella were enriched, while SMB53 was depleted in the HFrEF patients. Further, the authors performed virtual bacterial genome analyses to assess the metabolic pathway information carried by the gut microbiome. The predictive functional metagenomics using PICRUSt ${ }^{5}$ revealed several differences in bacterial genes associated with metabolic pathways in amino acid, carbohydrate, vitamin, and xenobiotic metabolism between the HFrEF patients and non-HF controls. ${ }^{4}$ However, we have to carefully interpret the data, because this is both virtual and genomic (bacterial DNA level) information, but neither functional protein levels nor metabolites are given. To obtain accurate information, one should perform whole bacterial genome analyses using shotgun sequence and metabolome analyses.

Previous investigations using 16S rRNA gene amplicon sequencing in hospitalized HF patients have reported compositional alterations of the gut microbiome relative to healthy or community-based controls. ${ }^{6}, \mathbf{7}$ Kamo et al found that the genera Clostridium and Dorea were less abundant in Japanese HFrEF patients younger than 60 years of age in comparison with age-matched healthy volunteers. ${ }^{6}$ In a population of European HFrEF patients, the genera Blautia, Collinsella, Erysipelotrichaceae, and Ruminococcaceae were decreased in HF patients compared with age- and sex-matched control subjects. ${ }^{7}$ Some meaningful conclusions could be drawn, but these observational studies were fraught with limitations. Firstly, the composition of the gut microbiome in HF patients was compared with that in controls with considerably different comorbidities and drugs from the patients with HF, who can be affected by not only the hemodynamic state of HF but also comorbidities including diabetes mellitus and hypertension, and medication use as well as diet. ${ }^{8}$ Secondly, there were some differences in stool collection and storage protocols between the HF patients and controls, which may have influenced the analytic results of gut microbial composition. ${ }^{9}$

In recent years, the challenge for microbiome research is to move from previous studies that merely define microbedisease associations to those that elucidate the cause-effect relationship. A mechanistic link between gut microbedependent generation of trimethylamine- $N$-oxide (TMAO) derived from specific dietary nutrients such as choline and carnitine and future cardiovascular events is well recognized. ${ }^{10}$ TMAO is formed in the liver from trimethylamine (TMA), which is produced by the gut microbial enzymes TMA lyases. Elevated TMAO levels have been directly linked to poor outcomes in patients with both acute and chronic HF. ${ }^{\mathbf{1 1}, 12}$ Different gut microbiome composition has varying abilities to generate TMAO; ${ }^{13}$ thus higher levels of circulating TMAO and increased cardiovascular disease risk can be attributable to a TMA-producing microbiome harboring TMA lyases. Other microbe-generated uremic toxins, such as indoxyl sulfate, have been reported to cause numerous harmful effects on the heart and kidney, and may play an important role in the development of HF (Figure). ${ }^{\mathbf{1 4}}$

In summary, recent data on the potential interaction between the gut microbiome and $\mathrm{HF}$ are intriguing. Morphological and functional alterations in the intestines or bacterial endotoxemia can be affected by inflammation, bowel ischemia, and/or congestion depending on HF severity. ${ }^{15}$ Studies have mainly focused on HFrEF patients, so the association between HF with preserved EF and the gut microbiome remains uncertain, and is an important topic to be clarified. With increasing awareness of the relationship between the gut microbiome and cardiovascular diseases, gut microbiome-derived metabolites such as TMAO and indoxyl sulfate and the specific bacteria capable of producing these uremic toxins are potential therapeutic targets. Further studies, focusing on a greater mechanistic understanding of the gut microbiome in the pathogenesis of $\mathrm{HF}$, are unquestionably necessary to develop novel diagnostic and therapeutic strategies for HF.

\section{References}

1. Go AS, Mozaffarian D, Roger VL, Benjamin EJ, Berry JD, Blaha MJ, et al. Heart disease and stroke statistics: 2014 update: A report from the American Heart Association. Circulation 2014; 129: e28-e29.

2. Marcinkiewicz-Siemion M, Ciborowski M, Kretowski A, Musial WJ, Kaminski KA. Metabolomics: A wide-open door to personalized treatment in chronic heart failure? Int J Cardiol 2016; 219: $156-163$.

3. Sonnenburg JL, Backhed F. Diet-microbiota interactions as moderators of human metabolism. Nature 2016; 535: 56-64.

4. Katsimichas T, Ohtani T, Motooka D, Tsukamoto Y, Kioka H, Nakamoto K, et al. Non-ischemic heart failure with reduced ejection fraction is associated with altered intestinal microbiota. Circ J 2018; 82: 1640-1650.

5. Langille MG, Zaneveld J, Caporaso JG, McDonald D, Knights D, Reyes JA, et al. Predictive functional profiling of microbial communities using 16S rRNA marker gene sequences. Nat Biotechnol 2013; 31: 814-821.

6. Kamo T, Akazawa H, Suda W, Saga-Kamo A, Shimizu Y, Yagi $\mathrm{H}$, et al. Dysbiosis and compositional alterations with aging in the gut microbiota of patients with heart failure. PLoS One 2017; 12: $\mathrm{e} 0174099$.

7. Luedde M, Winkler T, Heinsen FA, Rühlemann MC, Spehlmann ME, Bajrovic A, et al. Heart failure is associated with depletion of core intestinal microbiota. ESC Heart Fail 2017; 4: 282-290.

8. Falony G, Joossens M, Vieira-Silva S, Wang J, Darzi Y, Faust $\mathrm{K}$, et al. Population-level analysis of gut microbiome variation. Science 2016; 352: 560-564.

9. Gorzelak MA, Gill SK, Tasnim N, Ahmadi-Vand Z, Jay M, Gibson DL. Methods for Improving human gut microbiome data by reducing variability through sample processing and storage of stool. PLoS One 2015; 10: e0134802.

10. Heianza Y, Ma W, Manson JE, Rexrode KM, Qi L. Gut microbiota metabolites and risk of major adverse cardiovascular disease events and death: A systematic review and meta-analysis 
of prospective studies. J Am Heart Assoc 2017; 6: e004947.

11. Tang WH, Wang Z, Fan Y, Levison B, Hazen JE, Donahue LM, et al. Prognostic value of elevated levels of intestinal microbegenerated metabolite trimethylamine-N-oxide in patients with heart failure: Refining the gut hypothesis. J Am Coll Cardiol 2014; 64: 1908-1914.

12. Suzuki T, Heaney LM, Bhandari SS, Jones DJ, Ng LL. Trimethylamine N-oxide and prognosis in acute heart failure. Heart 2016; 102: $841-848$.

13. Cho CE, Taesuwan S, Malysheva OV, Bender E, Tulchinsky NF,
Yan J, et al. Trimethylamine-N-oxide (TMAO) response to animal source foods varies among healthy young men and is influenced by their gut microbiota composition: A randomized controlled trial. Mol Nutr Food Res, doi:10.1002/mnfr.201600324.

14. Gao H, Liu S. Role of uremic toxin indoxyl sulfate in the progression of cardiovascular disease. Life Sci 2017; 185: 23-29.

15. Sandek A, Bjarnason I, Volk HD, Crane R, Meddings JB, Niebauer J, et al. Studies on bacterial endotoxin and intestinal absorption function in patients with chronic heart failure. Int $J$ Cardiol 2012; 157: 80-85. 